were determined by high-resolution transmission electron microscopy to be conformal, uniform, continuous, and dense, with a controllable thickness between a few Ångstroms and a few nanometers. After drying the samples for $4 \mathrm{~h}$ at $150^{\circ} \mathrm{C}$, the team then used them to fabricate DSSCs. While niobia coatings did not improve the cell performance, cells based on electrodes with amorphous titania coatings were up to $65 \%$ more efficient than non-coated reference cells, and bi-layer titania/magnesia coatings led to up to $113 \%$ efficiency gains. The researchers attributed the enhancements to improved inter-particle connections from the titania layer and reduced recombination rates due to the insulating properties of the magnesia layer.

These results suggest that enhanced DSSCs fabricated by a low-cost, lowtemperature technique may one day offer a competitive alternative to conventional silicon photovoltaics.

COLIN MCCORMICK

\section{High Modulus Fluorinated Molding Materials Improve Fidelity in Soft Lithography}

Molding materials used for soft lithography, a nanofabrication technique, just got a little tougher. Polydimethylsiloxane (PDMS) elastomers, typically used as a molding material to replicate feature sizes greater than $100 \mathrm{~nm}$, tend to have a low elastic modulus (2.0 MPa) and low surface energy $(\sim 25 \mathrm{mN} / \mathrm{m})$. These properties make it easy to remove the PDMS from delicate master templates. New findings, however, show that increasing a material's elastic modulus may help improve pattern transfer fidelity, especially when replicating high aspect ratio features with small spacing or periodicity. A research team including S.S. Williams and coresearchers at the University of North Carolina at Chapel Hill, S. Retterer at Oak Ridge National Laboratory, R. Ruiz of Hitachi Global Storage Technologies, and J.M. DeSimone at the University of North Carolina at Chapel Hill and North Carolina State University reported in the April 14 issue of Nano Letters (DOI: $10.1021 /$ nl100326q; p. 1421) a composite molding technique that utilizes perfluoropolyether-(PFPE)-based elastomers. Williams and colleagues found that by increasing the modulus of the PFPE, smaller feature sizes could be replicated than when molds were made with PDMS.
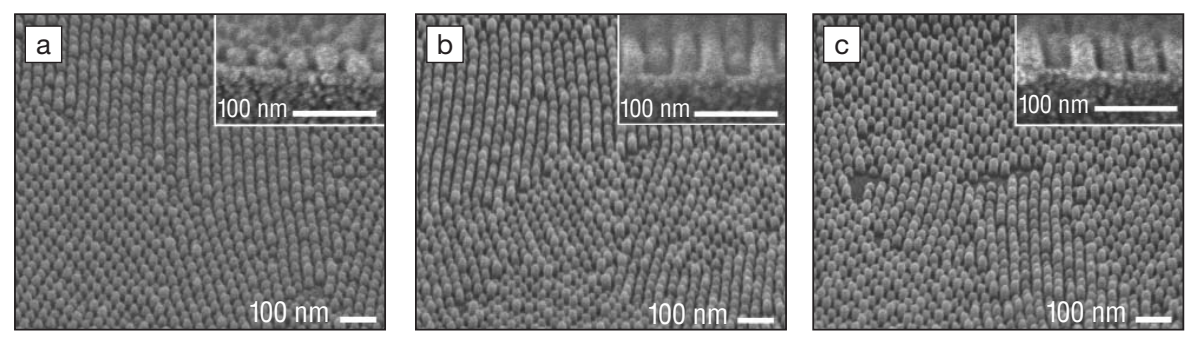

Figure 1. Trimethylolpropyl triacrylate (TMPTA) patterns formed using a variety of composite molds. TMPTA patterns made with molds composed of a low modulus material $(1.4 \mathrm{~K} \mathrm{per-}$ fluoropolyether, PFPE) (a) demonstrated a loss in resolution while TMPTA patterns from molds made with higher modulus materials such as (b) 80:201.4 K PFPE/TMPTA blend and (c) PFPE-tetramethacrylate showed much higher fidelity.

"The key to high fidelity molding materials," said Williams, "is that you need a combination of a high modulus material that retains a low surface energy." The team systematically examined the macromonomer functionality (i.e., the number of acrylate groups per polymer chain), the molecular weight of the macromonomer, and the addition of a multifunctional crosslinking additive (trimethylolpropyl triacrylate, TMPTA). Molds with higher functionality, lower molecular weight macromonomers, or TMPTA partially substituted for PFPE showed dramatic improvements in modulus with an insignificant bump in surface energy (see Figure 1). The molds with higher modulus had lower propensity for lateral collapse of high aspect ratio features.

Overall, the new PFPE molds improved fidelity of pattern transfer from the master to the mold and from the mold to TMPTA replicates. While previously reported PDMS molds were able to replicate $80 \mathrm{~nm} \times 80 \mathrm{~nm}$ feature sizes with $100 \mathrm{~nm}$ heights, the PFPE molds were able to replicate $20 \mathrm{~nm} \times 20 \mathrm{~nm}$ feature sizes with $40 \mathrm{~nm}$ heights in TMPTA. The researchers also note the ability to easily tailor the height of the flash layer, a feat that is difficult to obtain without the use of expensive equipment.

KEVIN P. HERLIHY

\section{Magnetic Field Applied to 3D Manipulation of Alloys}

The relentless drive toward increased miniaturization and better performance of microelectronic devices has necessitated three-dimensional (3D) architectures with vertical geometries. Such 3D architectures require stacked two-dimensional layers formed by photolithography or perpendicular vias for the conductive pathways. Various new approaches have been proposed to obtain such structures, including self-assembly and ink-jet printing; however, these use processes and materials not commonly found in current microelectronics fabrication facilities.

A different issue is the current use of lead-based solders as joining materials, serving as interconnects in devices. Due to serious health concerns associated with the use of lead-based solders, lead-free alternatives are being explored, such as $\mathrm{Sn}-3.5 \% \mathrm{Ag}$, and are being mandated through regulations such as the $\mathrm{Re}$ strictions on Hazardous Substances (RoHS). Unfortunately, this lead-free material has processing temperatures $30-40^{\circ} \mathrm{C}$ above the melting point of $220^{\circ} \mathrm{C}$, of the incumbent $\mathrm{SnPb}$ alloy, which can significantly affect device performance. Now, A.G. Ramirez and her co-workers at Yale University report in the March 16 issue of the Proceedings of the National Academy of Sciences (DOI: 10.1073/ pnas.1001410107; p. 4834) the development of tin-silver-based alloys containing iron dispersions that can be inductively heated and remotely manipulated in 3D space using a magnetic field. The iron dispersions allow for magnetically driven inductive non-contact heating, while at the same time imparting mechanical strength. 
In addition, they allow for remote manipulation through the same magnetic field (see Figure 1).

The alloys were prepared by incorporating multidomain magnetic dispersions of Fe particles (diameters under $44 \mu \mathrm{m}$ ) into Sn-3.5\%Ag by crushing, mixing, and then heating. A homogeneous distribution was achieved. The magnetic properties of the Fe dispersions were not affected at the melting point of $\mathrm{Sn}-3.5 \% \mathrm{Ag}$ since the Curie temperature of $\mathrm{Fe}$ is $770^{\circ} \mathrm{C}$. Various structures using these materials were constructed by applying a magnetic field and drawing the molten solder through holes in silicon wafers. Molten alloy was also drawn into channels of a polydimethylsiloxane mold. The shapes were released at room temperature and maintained.

The mechanical properties of the materials were measured at room temperature. The ultimate tensile strength of the alloys with the dispersions increased by nearly $40 \%$ over that of the dispersion-free material, though the ductility decreased. Elastic modulus increased monotonically with increasing amount of dispersion. The increase in strength with increased dispersion concentration was attributed to dispersion hardening. The strength was further enhanced by aligning the dispersions during the casting using a magnetic field. In a sample with 5\% iron, strength increased by $20 \%$ when cast under a magnetic field. It was determined that in the aligned materials, a composite structure of particle-rich hard layers and particledepleted soft layers formed. By a contactstrengthening mechanism, the hard layers delayed plastic deformation in the soft layers which improved overall strength.

The inclusion of magnetic dispersions did not change the electrical resistivity and melting point of the material, which is critical for use as effective interconnects. This was true of the vertical connections as well, which is necessary for 3D device architectures. The inclusion of the dispersions thus had little effect on these properties. The wettability of the alloy to a surface is crucial for electrical contact, and was found to be unchanged for the $\mathrm{Sn}-\mathrm{Ag}$ alloy for dispersion concentrations of up to $10 \%$ Fe. Above this, the wetting angle increased monotonically.

Induction heating of the material under a magnetic field due to the presence of the magnetic dispersions was one of the goals of this study. The issue of concern for the use of Sn-Ag alloys as solders to replace
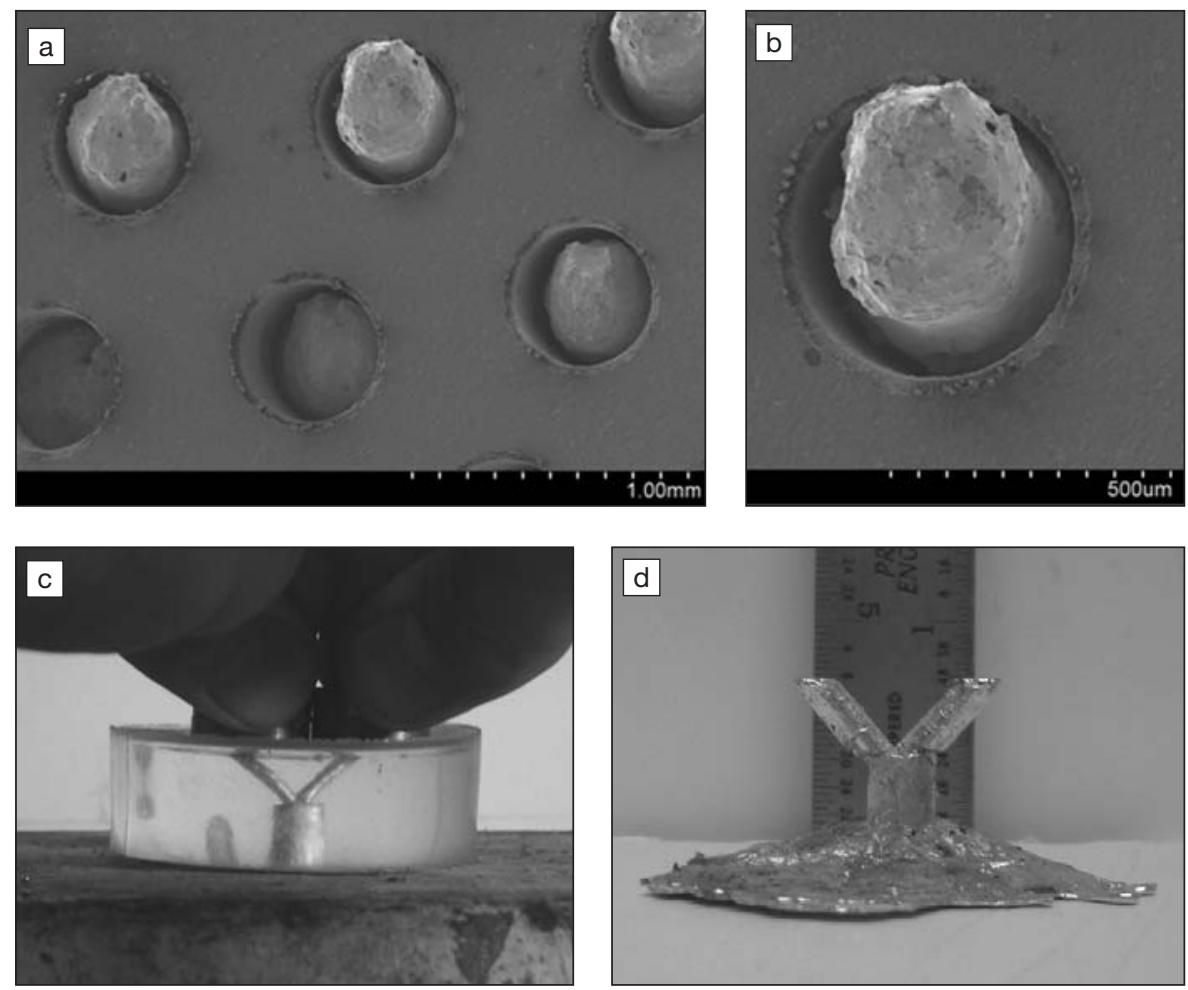

Figure 1. An array of structures made with magnetically responsive solders. (a) Scanning electron micrograph of silicon vias that have been filled with these materials. (b) One of the vias at a higher magnification. (c) A three-dimensional structure rapidly generated with a magnetically responsive alloy in a polydimethylsiloxane (PDMS) mold. Molten alloy was drawn from a reservoir into a Y-shaped cavity within PDMS using samarium cobalt magnets. (d) The extracted Y-shape after solidification of the alloy. Reproduced with permission from Proc. Natl. Acad. Sci. 107(11)(2010) 4834; DOI: 10.1073/pnas.1001410107. @2010 National Academy of Sciences.

lead-based solders is the higher melting temperature of the Sn-Ag system. This study showed that incorporating the magnetic dispersions resulted in induction heating, and also a faster rate of heating than the dispersion-free material. Thus, the dispersion-containing solder was hotter internally and could be easily deformed with pressure. This localized heating due to magnetic induction balanced out the higher melting temperature of the $\mathrm{Sn}-\mathrm{Ag}$ alloy. The researchers are in the process of determining the optimal concentration of the dispersion and the optimal magnetic field to determine the balance between heating efficiency and viscosity.

The present study demonstrated that materials can be magnetically heated and manipulated in 3D space. The versatility of the technique was shown by using it to create various devices, including a mag- netic-sensor circuit that creates an open circuit on application of a magnetic field and liquid conveyance of objects in a magnetically responsive molten metal. The researchers envision other applications such as self-healing assemblies and selfrepairing joints by application of a magnetic field. However, the primary motivation of the study was to demonstrate robust assembly of 3D interconnects, which the researchers accomplished. The dispersion containing Sn-Ag alloys could be heated using a magnetic induction without damaging adjacent components in microelectronic devices. The study also opens the door for the use of lead-free solders in devices without compromising mechanical properties, in accordance with increasing restrictions on the use of leadbased alloys.

GOPAL R. RAO

\section{www.mrs.org}

